\title{
Autophagy Mechanism, Regulation, Functions, and Disorders
}

\author{
Mallikarjun Badadani \\ Department of Pediatrics, University of California at Irvine, 2501 Hewitt Hall, Irvine, CA 92697, USA \\ Correspondence should be addressed to Mallikarjun Badadani, badadani@gmail.com
}

Received 31 May 2012; Accepted 30 July 2012

Academic Editors: D. Arnoult, D. Bouvard, and A. Chiarini

Copyright ( 2012 Mallikarjun Badadani. This is an open access article distributed under the Creative Commons Attribution License, which permits unrestricted use, distribution, and reproduction in any medium, provided the original work is properly cited.

\begin{abstract}
Autophagy is a self-digesting mechanism responsible for removal of damaged organelles, malformed proteins during biosynthesis, and nonfunctional long-lived proteins by lysosome. Autophagy has been divided into three general types depending on the mechanism by which intracellular materials are delivered into lysosome for degradation that is, microautophagy, chaperonemediated autophagy (CMA), and macroautophagy. In microautophagy cytoplasm material is sequestered through direct invagination to the lysosomal membrane. Whereas in CMA proteins flagged with pentapeptide motif (KFERQ) were selectively degraded through direct translocation into lysosome. Macroautophagy involves the formation of subcellular double-membranebound structures called autophagosomes that contain degradable contents of cytoplasm materials and deliver them into lysosomes for breakdown by lysosomal enzymes. The molecular mechanism of autophagy involves several conserved Atg (autophagy-related) proteins. Systems produce modified complexes Atg8-PE and Atg5-Atg12-Atg16 as autophagy regulators. Autophagy is activated in response to diverse stress and physiological conditions. For example, food deprivation, hyperthermia, and hypoxia are mediated by factors like insulin/IGF-1, m-TOR signaling, FOXO transcription factors, and chaperones. The perturbance in autophagy may lead to several types of cancers, myopathies, and neuromuscular disorders. Several autophagy inducers and inhibitors like 3methyladenine (3-MA), bafilomycin A1, LY294002 (LY), and Velcade have been used to treat disease is an intense field of study.
\end{abstract}

\section{What Is Autophagy?}

Before delving into the aspects of autophagy, There ia a wellknown answer to the question: What is an autophagy? In the current science, autophagy is a self-digesting mechanism responsible for removal of long-lived proteins, damaged organelles, and malformed proteins during biosynthesis by lysosome [1-3]. The evidence demonstrates that autophagic process is meant for regulating diverse cellular functions including growth, differentiation, response to nutrient deficit and oxidative stress, cell death, and macromolecule and organelle turnover.

\section{Autophagy Mechanism}

Autophagy involves the formation of a double-membrane vesicle, which encapsulates cytoplasm, malformed proteins, long-lived proteins, and organelles and then fuses with lysosomes for degradation. The formation of the doublemembrane vesicle is a complex process involving 16 autophagy-related proteins (Atg proteins). Apart from this, two ubiquitin-like conjugation systems are involved in autophagy. These systems produce modified complexes of autophagy regulators: Atg8-PE and Atg5-Atg12-Atg16, and that may determine the formation and size of the autophagosome. The nucleation, expansion, uncoating, and completion of the autophagosome formation then occur, leading it to fuse with lysosomes [4].

The molecular mechanism of autophagy involves several conserved Atg (autophagy-related) proteins, most of which were first identified in yeast [5-7]. Initiation of autophagosome formation requires two complexes. (1) A complex that contains the class III PI3 K Vps34, Atg6/Beclin1, Atg14, and Vps15/p150.73. (2) The other complex includes a serine/threonine kinase Atg1. The kinase activity of Atg1 requires the function of two other autophagy proteins, that is, Atg13 or Atg8 and Atg17. In mammals, which do not contain Atg13, Atg1 was found to associate with the Atg8 orthologues, LC3 (microtubule-associated protein light chain 3), GATE-16 (Golgi-associated ATPase enhancer of 
$16 \mathrm{KDa}$ ), and GABARAP (G-amino butyric acid type A receptor-associated protein).

The elongation involves two ubiquitin-like conjugation pathways, the Atg8/MAP-LC3/GABARAP/GATE-16 and Atg12 systems. The soluble Atg8 protein undergoes a carboxyl-terminal cleavage by the cysteine protease Atg4 to expose a reactive glycine residue to mediate autophagosome formation. Atg4 is activated by the Atg7 (E1like) and Atg3 (E2-like) enzymes. The function of Atg3 requires a protein complex which involves Atg5, Atg12, and Atg16. The phosphatidyl ethanolamine is then covalently bound to activated Atg8 (Atg8-PE in yeast and lipidated LC3-II in mammals). It remains bound to the autophagosome membrane until some of it was cleaved by Atg 4 to be recycled. If uncleaved by Atg 4 it gets degraded within the autolysosome, as Atg8 remains covalently bonded to the membrane and may therefore be used as a marker for autophagy. As soon as autophagosome formation is completed, the Atg16-Atg5-Atg12 complex dissociates from its bordering membrane, and components take part in a recycling process mediated by Atg2, Atg9, and Atg18. Now the completed autophagosome is ready for fusion with the endosome or the lysosome.

\section{Autophagy Regulation}

Autophagy is activated in response to diverse stress and physiological conditions. For example, food deprivation, hyperthermia, and hypoxia, which are known as major environmental modulators of ageing, are also conditions that induce autophagy [8-10]. At molecular level, an autophagy pathway displays remarkable interrelations with factors influencing ageing.

\section{Insulin/IGF-1 Regulates Autophagy}

The nutrient-responsive IGF-1/Insulin signaling pathway promotes reproductive growth, morphogenesis, and survival. In C. elegans, insulin/IGF-1 signaling pathway prevents dauer development. In mutants, dauer development is independent of growth conditions like reduced insulin/IGF1 receptor activity. The mutant animals were reported to show high level of autophagy in the cells associated with morphogenesis. The disruption in autophagy gene function inhibits morphogenesis and survival. Certain autophagy genes have been reported to influence on cell growth, the effects being mediated by insulin/IGF-1 or TGF $\beta$ signaling. For example, nematodes are unable to grow in giant body size phenotype when autophagy gene activity was disrupted and with aberrant insulin/IGF1 or TGF $\beta$ receptor activity. This implies that autophagy genes are controlling the cell size converged by the growth modulator signaling pathways $[11,12]$. In mammals, the relationship between autophagy and insulin/IGF-1 signaling appears to be more intimate.

\section{5. m-TOR Regulates Autophagy}

Nutrient deprivation or treatment with rapamycin induces autophagy. In yeast, Atg13 is hyperphosphorylated by TORC1 (the rapamycin-sensitive TOR kinase complex 1) and has lower affinity for Atg1 [13]. Apart from this, TORC1 controls activation of several effectors that regulate transcription or translation of certain proteins by phosphorylation, some of which are required for autophagy. TOR regulates induction of autophagy in cooperation with two other nutrient sensing pathways, that is, protein kinase A and SCh9 [14]. In mammals, mTOR appears to regulate autophagy in similar way to the yeast. Further, AMPK acting at upstream of TOR promotes autophagy in human cell lines and flies [15]. Thus, mTOR along with insulin/IGF1 signaling controls autophagy at several levels. Normal cell growth requires a well balance between protein/organelle synthesis and degradation (turnover). Increased growth rate implies accumulation of aberrant cytosolic components. Irreversibly modified (e.g., oxidised, crosslinked) proteins, malformed proteins, and defective mitochondria act as cellular toxins, which interfere with normal cellular function. These cellular toxins accumulate progressively as the age is advanced, providing a gradually reduced ability of the organisms, normal functions and to survive [16-19].

The evidence shows that mammalian target of rapamycin (mTOR) acts as a negative regulator, and the extent of autophagy is regulated by proteins upstream of mTOR signaling, including PTEN, PDK1, Akt, and TSC1/2 [20]. For example, PTEN and TSC1/2 positively regulate autophagy, whereas Akt inhibits it. Downstream targets of mTOR, including elongation factor-2 kinase [21] and S6kinase [20], have been shown to regulate autophagy.

\section{DRAM and p53 Regulate Autophagy}

The fact is that p53 suppressor is mutated in approximately $50 \%$ of human cancers and induces autophagy. It has been reported that P53 functions as critical mediator for damage-induced apoptosis and has been shown to induce autophagy in DRAM- (Damage-regulated autophagy modulator-) dependent manner to execute a full cell death in human cancer cell lines. DRAM is a lysosomal integral membrane protein, and a direct target of p53-induces macroautophagy and helps in accumulation of autophagosomes $[21,22]$. The p53 mediated apoptosis involves several chromatin-remodeling factors [23] (e.g., e2f1 as it involves in transcriptional repression of cell proliferation as part of component with retinoblastoma complex). Autophagy genes may also be controlled by the factors involved in chromatin remodeling as p53 functions at upstream of autophagy pathway to cause cell death [7]. Recent data show that e2f1 binds to Atg1, atg8, and DRAM regulatory regions in human [24].

\section{FOXO and ROS Regulate Autophagy}

Recently, it has been reported that transcriptional level of several autophagy-related genes has been increased by 
activated FOXO. The genes included were Atg8/LC3, Atg12, Vps34, and Atg6 to induce protein degradation in atrophying muscle cells, and surprisingly the regulatory effect of FOXO on Atg8 and Atg12 seems to be direct [25]. The chromatinremodelling factors deacetylate FOXO transcription factors and promote longevity in worms, flies, and mammals [2629]. Recent evidence demonstrates that in mice, sirtuin-type Sirt1 is able to induce autophagy in both normal growth and starvation conditions. Sirt1 acetylates Atg5, Atg7, and Atg8 autophagy factors in an NADP-dependent manner [30]. The loss of function of Sirt1 in mutant phenotype resembles those of autophagy-defective mice, such as early death and the accumulation of abnormal mitochondria.

Reactive oxygen species (ROS) also regulate starvationinduced autophagy. Recent evidence shows that Atg4, an essential protease in the autophagy machinery, has been recognized as a direct target for oxidation by ROS. Accumulation of ROS generated during various cellular activities mainly by respiration causes an oxidative stress. The cells respond to oxidative stress by activating various defense mechanisms. Several studies show that ROS act as signaling molecules and are able to induce the autophagic process and cause subsequent loss of the affected cells. Autophagy has a critical function in the cellular response to oxidative stress [31].

\section{Chaperones}

Autophagy is a lysosome-mediated degradative process of eukaryotic cells to digest their own constituents during development or starvation [32-34]. The autophagy has been divided into three general types depending on the mechanism by which intracellular materials are delivered into the lysosome for degradative process: (1) microautophagy, (2) chaperone-mediated autophagy (CMA), and (3) macroautophagy. In microautophagy, cytoplasmic material is sequestered through direct invagination to the lysosomal membrane. In chaperone-mediated autophagy (CMA), proteins bearing a particular pentapeptide motif (KFERQ) are selectively degraded through direct translocation into the lysosome. This is absent in plants. The overall process of autophagy has been shown schematically in Figure 1.

Macroautophagy involves the formation of subcellular double-membrane-bound structures called autophagosomes, contains degradable contents to sequester cytoplasmic materials, and delivers them into lysosomes for breakdown by lysosomal enzymes.

The products of degradation can be reused for cell functioning. The process of macroautophagy (hereafter referred to as autophagy) starts with the initiation of the formation of the isolation membrane (phagophore), a process called nucleation. The growth of the phagophore (elongation or expansion) terminates in the completion of the autophagosome. Then the fusion of the autophagosome with lysosome forms an autolysosome within which the enclosed material is degraded $[6,7,35]$.

Chaperone-mediated autophagy (CMA) is differentiated from other types of autophagy by two properties, that is, its selectivity towards a particular pool of cytosolic proteins and the mechanism of delivery of the substrate proteins to lysosomes. It is estimated that about $30 \%$ of soluble cytosolic proteins labeled with CMA target motif pentapeptide KFERQ. Only proteins bearing this particular targeting motif in their amino acid sequence are selectively recognized by the heat shock cognate protein of $70 \mathrm{kDa}$ (hsc70), the chaperone that mediates their delivery to lysosomes [32-34]. Some of the following are identified as substrate proteins that include glycolytic enzymes (glyceraldehyde-3-phosphate dehydrogenase, aldolase, and phosphoglyceromutase), $20 \mathrm{~S}$ proteasome, transcription factors and inhibitors of transcription factors (c-fos, the inhibitor of $\mathrm{NF} \kappa \mathrm{B}[\mathrm{I} \kappa \mathrm{B}]$ ), $\alpha 2$ microglobulin, calcium-binding proteins (Annexins I, II, IV, and VI), and proteins associated to vesicular trafficking $(\alpha-$ synuclein) $[32,36]$.

After the CMA targeting motif is recognized by hsc70, the substrate/chaperone complex is targeted to the surface of the lysosomes where it binds to the lysosome-associated membrane protein type 2A (LAMP-2A), a receptor for CMA [37]. Once bound, the substrate unfolds [38] and crosses the lysosomal membrane assisted by a lysosomal form of hsc70 (lys-hsc70) present in the lumen [39]. CMA substrates access the lysosomal lumen directly across the lysosomal membrane. Transport is saturable which requires a source of energy (ATP) and is temperature dependent (binding occurs at temperatures as low as $10^{\circ} \mathrm{C}$, but transport is only detected at temperatures above $\left.25^{\circ} \mathrm{C}\right)[40,41]$.

The selectivity to degrade associated through CMA seems beneficial under specific conditions in which discrimination is required for different types of proteins. During prolonged starvation, the CMA provides the amino acids required for protein synthesis but also favors degradation of unnecessary proteins against that of proteins essential for cell survival $[42,43]$. Similarly, during mild oxidative stress [44] or after exposure to protein-modifying toxic compounds [45], this allows the selective removal of the proteins damaged or altered under these conditions.

\section{Autophagy and Cancer}

The cell death alteration in cancer is one of the hallmarks, and these cells are under the pressure of survival. The unsuccess in apoptosis causes these cells to transform and leads to genetic damage and carcinogenesis $[46,47]$. There are so much information about mutational and expressional alterations of apoptosis genes, such as Fas and caspases in human cancers [48-51]. However, data on autophagy genes is more limited than apoptosis [52-54]. In cancers, ATG2, ATG5, ATG9, ATG12, and UVRAG genes have been reported to be mutated with microsatellite instability [55]. The Atg6 (Beclin1) gene is deleted in some cancers [55], and rarely point mutation is found in human cancers [54]. The UVRAG mutated binds with Beclin-1 in gastric and colorectal cancers with microsatellite instability [53]. The antiapoptosis genes are likely to be potential oncogenes, while procell death genes are likely to be potential tumor suppressor genes. There is controversy about the role of 


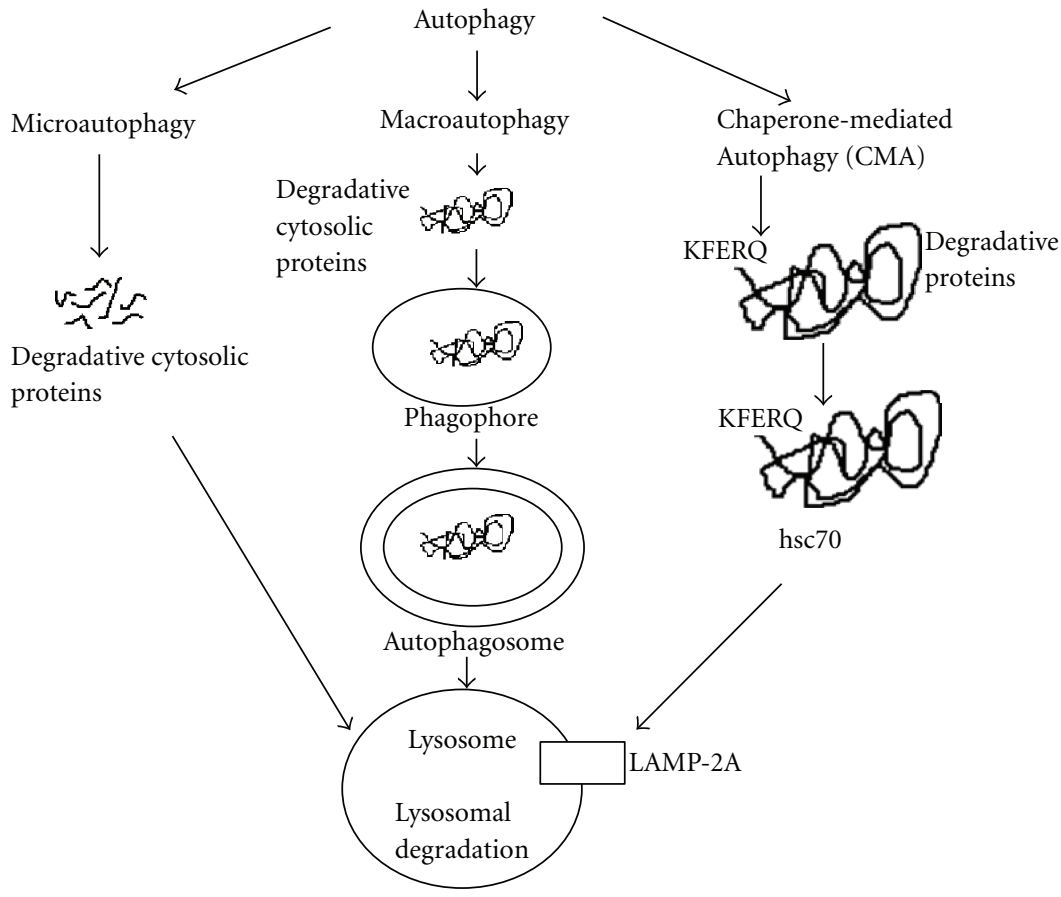

FIGURE 1: The different types of autophagy processes for degradation of targeted proteins and unwanted cellular organelles.

autophagy in cancer. Some data favor the idea that autophagy suppresses tumorigenesis, whereas other data suggest that autophagy enhances tumorigenesis and protects tumor cells from cell death [56].

When primary epithelial cells become immortal, a cell death pathway that involves both autophagy and apoptosis is selectively inactivated [57]. In model systems of mammary acini formation, both apoptosis and autophagy are involved in the removal of epithelial cells to form luminal structures [58]. This suggests that autophagy prevents early steps in epithelial tumor development. Taken together, these data imply that autophagy can both stimulate and prevent cancer depending on the context. To further test this idea, it is much more interesting to study mice with defects in other Atg genes to see if they also have a cancer predisposition phenotype similar to the Beclin- $1^{+/-}$mice.

ATG5 is a protein involved in the early stage of autophagosome formation [59, 60]. Binding of ATG5 with ATG12 contributes to autophagosome formation, which sequesters cytoplasmic materials before lysosomal delivery [61]. Apoptotic stimuli cleave ATG5 which is subsequently translocated to mitochondria and triggers caspase activation. Expression of ATG5 sensitizes tumor cells to chemotherapy, but silencing of ATG5 results in a resistance to the chemotherapy [62].

The roles of autophagy in cancer are a topic of intense debate. As mentioned above, autophagy allows a cell to respond to changing environmental conditions, such as nutrient deprivation. On starvation, autophagy is greatly increased, allowing the cell to degrade proteins and organelles and thus obtain a source of macromolecular precursors, such as amino acids, fatty acids, and nucleotides, which would not be available otherwise. Thus, autophagy serves as protective role allowing cells to survive during nutrient deprivation.

Moreover, when autophagy is prevented under these conditions, cells undergo apoptosis $[63,64]$. Thus, when tumor cells are starved, autophagy stops them from dying by inhibiting apoptosis. In a tumor, this may mean that autophagy keeps tumor cells alive when limited angiogenesis leads to nutrient deprivation and hypoxia. Therefore, we would expect that increased autophagy would promote the growth of solid tumors, whereas reduced autophagy might provide a useful way to limit tumor growth. In stark contrast to this potential cancer-promoting effect of autophagy, numerous lines of evidence indicate an anticancer role. The autophagy gene Beclin-1 (the mammalian counterpart of the yeast Atg6 gene), which is part of a type III phosphatidyl inositol 3-kinase complex required for autophagic vesicle formation, is a haploinsufficient tumor suppressor in mice $[65,66]$ and is monoallelically lost in human breast, ovarian, and other tumors [67]. Moreover, p53 and PTEN are two of the most commonly mutated tumor suppressor genes, and both induce autophagy $[68,69]$. Conversely, the oncogenic protein $\mathrm{Bcl}-2$ directly interacts with Beclin-1 to inhibit autophagy [70] because oncogenes can inhibit autophagy and tumor suppressors induce autophagy. Whereas a bona fide autophagy regulator is itself a tumor suppressor, these data suggest that autophagy serves as an anticancer role. The mechanism through which autophagy inhibits tumor development is unclear. Possibilities include limiting tumor cell growth or reducing mutagenesis or other damage 
caused by reactive oxygen species by removal of damaged mitochondria and other organelles. Alternatively, autophagy may kill developing tumor cells.

\section{Autophagy and Cell Death}

Programmed cell death can be divided into apoptotic (type I) and autophagic (type II) cell death. In addition, there may be forms of programmed necrosis and other even less welldefined death pathways. The molecular pathways involved in the regulation and execution of apoptosis have been well defined. But the mechanisms of autophagic cell death have not been studied well. Attempts to define autophagic cell death have been limited to morphologic characteristics, such as extensive autophagosomal/autolysosomal formation and Atg-8/LC3 translocation to autophagic vesicles. Recently, there are some reports that had defined one mechanism of autophagic cell death. These investigators showed that autophagic cell death caused by caspase inhibition is achieved through the selective autophagic degradation of catalase that leads to the generation of reactive oxygen species that kills the cell [71].

\section{Autophagy and Diseases}

There have been several recent reviews about autophagy as it relates to cancer and other diseases $[67,72]$. Autophagy is a genetically programmed, evolutionarily conserved process that degrades long-lived cellular proteins and organelles. Autophagy is important in normal development and responds to changing environmental stimuli. In addition to its role in cancer, it is important in numerous diseases, including bacterial and viral infections, neurodegenerative disorders, several myopathies, and cardiovascular diseases [72].

\section{Myopathies}

Neuronal tissues and skeletal muscles are main organs where autophagy is physiologically enhanced [73]. The accumulation of autophagic vacuoles is seen in skeletal myofibers in several neuromuscular disorders [74-78]. Based on the findings of vacuoles, these diseases are called autophagic vacuolar myopathies (AVMs). Two disorders belonging to this group have been associated with primary lysosomal protein defects, namely, Danon disease and Pompe disease [79].

\section{Danon Disease}

Danon disease is caused by the primary deficiency of a lysosomal membrane protein and lysosome-associated membrane protein-2 (LAMP-2), instead of a glycolytic enzyme [80]. Glycogen accumulation in fact is not a constant feature, and detailed pathological features are different from those of acid maltase deficiency. The name "glycogen storage disease IIb (GSDIIb)" should be discouraged as the earlier thought that the disease is due to maltase deficiency [81].
Danon disease is characterized by an X-linked dominant inheritance pattern, as a result of which males are more severely affected than females, although females develop symptoms at a later onset [82].

The causative protein, LAMP-2A, is a single-spanned membranous protein with molecular mass of $95-120 \mathrm{kDa}$. LAMP-2A was reported to function as a receptor for chaperone-mediated autophagy, in which the certain cytosolic proteins with a consensus sequence containing a sequence motif related to the pentapeptide KFERQ, such as ribonuclease A and glyceraldehydes 3-phosphate dehydrogenase, are selectively taken up and degraded in the lysosome $[83,84]$.

It has been demonstrated that LAMP-2A is required for the conversion of early autophagic vacuoles to vacuoles, indicating its involvement in the fusion of autophagic vacuoles with endosomes and lysosomes. LAMP 2-deficient mice exhibit elevated mortality after 20 days of age and show accumulation of autophagic vacuoles in liver, kidney, pancreas, and cardiac and skeletal muscles [85]. The failures in the normal progression of autophagic process in the absence of LAMP-2 have been presented by using cultured hepatocytes. These include accumulation of early autophagic vacuoles and intracellular mistargeting of lysosomal enzymes and LAMP1. Instead of the elevation of lysosomal enzyme secretion, these cultured hepatocytes show improper cathepsin D processing, abnormal retention of mannose-6-phosphate receptors in autophagic vacuoles, reduction of degradation of long-lived proteins, and nonsignificant induction of autophagic protein degradation after starvation.

\section{X-Linked Myopathy with Excessive Autophagy (XMEA)}

XMEA is a rare X-linked recessive AVM characterized by a slowly progressive weakness and atrophy of the proximal muscles [86]. Most patients maintain independent ambulation even beyond 60 years of age. Muscle pathology is similar to the findings seen in Danon disease. There are some features that are different from Danon disease, indicating that XMEA is a different myopathy. Other characteristic features include deposition of complement C5b-9 and calcium [87] on sarcolemma. The electron microscopy shows that the basal lamina appears multilayered [88] and that numerous exocytosed materials are within the basal lamina $[86,89]$. Till now, the gene responsible for XMEA has not been identified but mapped to the telomeric region of the long arm of chromosome X (Xq28). As XMEA has similarities in pathology with Danon, it has been assumed that the causative gene probably encodes a lysosomal protein.

\section{Pompe Disease (Acid Maltase Deficiency)}

Pompe disease (or glycogen storage disease type II) is an autosomal recessive disease due to primary deficiency of acid $\alpha$-1, 4-glucosidase which is also called acid maltase. It is lysosomal acid $\alpha$-glucosidase (GAA; EC 3.2.1.3), an exo-1,4- and -1,6- $\alpha$-glucosidase that specifically hydrolyzes glycogen to glucose. The GAA protein precursor has 952 
amino acids with a predicted molecular mass of $110 \mathrm{KDa}$. Furthermore, the newly synthesized precursor undergoes several steps of processing to give the 70 and $76 \mathrm{KDa}$ mature forms. More than 50 mutations have been reported in the gene encoding GAA, leading to a total or partial deficiency of lysosomal GAA. Importantly, among all enzymes responsible for glycogen storage disease, GAA is the only enzyme localized in the lysosomes, while all other enzymes are present in the cytosol. A lysosomal abnormality is seen only in Pompe disease among all glycogen storage diseases and is the prototypic lysosomal storage disorder [90, 91]. Clinically this disease is classified into two forms: infantile and late onset [92]. The late onset form is further divided into childhood, juvenile, and adult types. Nonsense mutations are more commonly seen in the infantile onset form. Deficiency of GAA leads to accumulation of lysosomal glycogen in virtually all cells of the body, but the effects are most notable in cardiac and skeletal muscles. Intracytoplasm vacuoles are prominent in the infantile form of acid maltase deficiency more than in the adult form. Characteristically, these vacuoles are so large that these occupy most of the space in many muscle fibers, often resulting in a "lace-like" appearance. Moreover, it has been observed that these vacuoles contain amorphous materials and are strongly stained with periodic acid Schiff staining, indicating that these are glycogen containing.

\section{Neuromuscular Disorders}

Neuromuscular disorders show accumulation of inclusion bodies apart from having vacuoles in their cells and have been classified as sporadic inclusion body myositis (sIBM). Sporadic inclusion body myositis (sIBM) is the most common progressive muscle disorder of older persons $[93,94]$. The characteristic features of sIBM muscle fibers are autophagic vacuoles, accumulation of ubiquitin-positive multiprotein aggregates containing misfolded proteins in the pleated sheet conformation of amyloid, and mononuclear cell inflammation $[95,96]$. In addition to their vacuolization, sIBM muscle fibers have other intriguing features, and their clinical spectrum includes phenotypic similarities to Alzheimer's disease and Parkinson's disease brains [94].

Malfunctioning of CMA has been described in familial forms of Parkinson's disease where mutated $\alpha$-synuclein is delivered to lysosomes for degradation via CMA. But after binding to LAMP-2A, it cannot be translocated to the lysosomal lumen resulting in blockage of this pathway [97]. During aging, CMA activity decreases due to a decrease in the levels of LAMP-2A at the lysosomal membrane [98], causing the accumulation of abnormal proteins leading to the higher stressors characteristic of old organisms [39, 98-101].

Vacuoles in sIBM muscle fibers are considered to be autophagic, because they often contain (i) lysosomal membranous debris by light and electron microscopy, which is considered a result of indigestibility of normally turned over or pathologically damaged cellular proteins and organelles, (ii) acid phosphatase positivity, and (iii) increased immunoreactivity of some lysosomal enzymes [102, 103]. Although the pathogenesis of sIBM seems complex and multifactorial, accumulations of the undegraded, misfolded proteins and putatively toxic oligomers have been proposed to be an important aspect. Recently, it has been reported the increased synthesis and accumulation of p62/SQSTM1 in muscle fibers and cultured human myocytes from sIBM. The p62 is ubiquitin-binding protein allegedly shuttling polyubiquitinated proteins for their degradation by both proteasome and lysosomes which have been demonstrated $[104,105]$. Increased accumulation of p62/SQSTM1 in sIBM muscle fibers is also an indicator of inadequate protein disposal system.

The two major pathways of cellular protein degradation relate to the $26 \mathrm{~S}$ proteasome and the autophagic/lysosomal systems. The $26 \mathrm{~S}$ proteasome, also called the ubiquitinproteasome system, is a major degradation mechanism for (i) normal regulatory and other short-lived proteins and (ii) misfolded proteins exported from the endoplasmic reticulum (ER) through a ubiquitinmediated ATP-independent process [106-108]. However, long-lived structural proteins, damaged or misfolded proteins, and obsolescent cellular organelles are degraded through autophagy [109-112]. Macroautophagy refers to formation and maturation of autophagosomes, which are structures carrying proteins and organelles destined for lysosomal degradation as discussed earlier. Abnormal accumulation within a cell of misfolded or damaged proteins which might occur due to either their excessive production, proteasome inhibition, oxidative stress, or other stressors increases the need for autophagic degradation accompanied by increased formation and maturation of autophagosomes [105, 107]. However, when lysosomal degradation is impaired or the amount of material to be degraded exceeds lysosomal capability, formation of autophagosomes dramatically increases, leading to formation of autophagic vacuoles. In mammalian cells, the presence of LC3-II protein on immunoblots, a lipidated form of the autophagosomal protein LC3 (microtubuleassociated protein light chain $3 \mathrm{~B}$ ), is considered the most important indicator of increased formation and maturation of autophagosomes [110, 112].

A recently found disease, inclusion body myopathy, Paget's disease of bone and fronto temporal dementia (IBMPFD), also shows similar pathology to sIBM in muscle fibres. The disease is due to mutation in p97/VCP (valosincontaining protein). VCP belongs to the family of type II ATPase associated with a variety of cellular activities (AAA) having two ATPase domains. It has been suggested to be involved in a number of cellular activities, including homotypic membrane fusion, transcription activation, nuclear envelope reconstruction, postmitotic organelle reassembly, cell cycle control, apoptosis, and endoplasmic reticulum associated degradation of proteins (ERAD). The pathology includes accumulation of inclusion bodies positive to ubiquitin and Tar-binding protein-43 (TDP-43). TDP-43 protein is evolutionary conserved, and its structure consists of a glycine-rich domain and two RNA recognition motifs. The exact function of TDP-43 remains unclear, though it is known to bind DNA and RNA (such as human immunodeficiency virus type 1 TAR DNA sequence motifs) and to be involved in the regulation of messenger RNA 
splicing and exon skipping. Increased LC3-II staining and TUNEL-positive nuclei have also been demonstrated. The clinical spectrum also includes Paget's disease of bone and frontotemporal dementia apart from inclusion body myopathy. These data suggest that IBMPFD may be due to impairment in autophagy and ER stress [113].

\section{Autophagy Diseases and Treatment}

Several drugs have been used to treat the autophagy-related disorders, but concurrent treatment has not yet arrived. Here it has been focused on the drugs alone or in combination to treated disease. The drugs have been used to either induce or block the autophagy pathway to alleviate the disease.

3-Methyladenine (3-MA) is an agent long known to be selective and potent inhibitor of autophagy-dependent protein degradation and suppresses the autophagosomes formation [114, 115]. Another agent, Bafilomycin A1 (Baf A1), acts to block the fusion of autophagosomes and lysosomes [116]. Both 3-MA and Baf Al could inhibit autophagy at doses of $10 \mathrm{mM}$ and $100 \mathrm{nM}$, respectively, in NB4 cells. The expressions of LC3 and Beclin1 both were decreased when treated with 3-MA. In Baf A1-treated cells, autophagy is inhibited before the fusion of autophagosomes and lysosomes. As a result, aggregates of LC3-II were found on the autophagosomes and cannot be degraded through the fusion of autophagosomes and lysosomes. Therefore, in these cells, the increase of LC3-II expression indicates that autophagy is suppressed.

Another drug LY294002 (LY) is inhibitor of Akt pathway. The LY has been used to study whether blocking of Akt pathway suppress the autophagy. EM results show that the formation of autophagosome decreased in LY-treated cells. In addition, Beclin-1 and LC3-II also decrease in NB4 cells. It implies that the Akt inhibition could suppress the autophagy [116].

Four p97 inhibitors have been reported till now. (1) Eeyarestatin I is the ERAD inhibitor found to directly associate with p97 and ER. It inhibits p97-associated deubiquitinating enzymes as well as p97-dependent protein degradation except ATPase activity. It has been demonstrated that p97-associated deubiquitination is involved in ERAD. (2) 2-Anilino-4-aryl-1, 3-thiazole compounds inhibited p97 ATPase activity and p97-associated protein degradation. (3) The Syk inhibitor III irreversibly inhibited p97 ATPase activity through its interaction with Cys522 within the D2 ATPase domain of p97 and the ubiquitin-fused reporter protein. (4) A chemical agent N2, N4-dibenzylquinazoline2, 4-diamine (DBeQ) was identified as a selective, potent, reversible, and ATP-competitive p97 inhibitor by screening a library of chemical compounds. DBeQ inhibited ERAD and autophagy and induced apoptosis by activating several caspases. $\mathrm{DBeQ}$ also showed antiproliferative activity on human cancer cells.

It has been demonstrated that inhibition of the ubiquitin-proteasome system and ER homeostasis may offer attractive therapeutic possibilities for certain types of human cancer. Thus, the development of p97 inhibitors has emerged as a therapeutic strategy for the treatment of human cancer. It is interesting to mention that expression level of p97 is correlated with progression, prognosis, and recurrence of certain types of cancer [117].

Many compounds that either interfere or block proteasomal function have been identified. These include $\beta$ lactones such as clastolactacystin, peptidyl aldehydes such as MG132 (carbobenzoxy-L-Leu-L-Leu-L-Leu), and peptidyl boronates such as Velcade (bortezomib/PS-341). Lactacystin binds covalently, whereas MG132 and Velcade bind reversibly to the N-terminal Thr residue of the $\beta 1$ subunit within the $26 \mathrm{~S}$ proteasome. Velcade (bortezomib/PS-341) is the proteosome inhibitor which has been shown to block the targeted proteolytic degradation of short-lived proteins that are involved in cell maintenance, growth, division, and death, advocating the use of proteasomal inhibitors as therapeutic agents. It has been demonstrated that the use of one proteasomal inhibitor for therapy and the combination of proteasome inhibitors Lactacystin and MG132 may be more effective in inducing apoptosis [118].

\section{Conclusion}

In summary, taken together the in-depth study of autophagy mechanism and regulation in cancer and its inducers and inhibitors to treat the disease is required. Similarly, several myopathies and neuromuscular disorders need to be focused in relation to autophagy and cell death. The study may be helpful in drug discovery and its application to alleviate the autophagy-related disorders.

\section{References}

[1] E. H. Baehrecke, "Autophagy: dual roles in life and death?" Nature Reviews Molecular Cell Biology, vol. 6, no. 6, pp. 505510, 2005.

[2] A. L. Edinger and C. B. Thompson, "Death by design: apoptosis, necrosis and autophagy," Current Opinion in Cell Biology, vol. 16, no. 6, pp. 663-669, 2004.

[3] Y. Kondo, T. Kanzawa, R. Sawaya, and S. Kondo, "The role of autophagy in cancer development and response to therapy," Nature Reviews Cancer, vol. 5, no. 9, pp. 726-734, 2005.

[4] T. Yorimitsu and D. J. Klionsky, "Autophagy: molecular machinery for self-eating," Cell Death and Differentiation, vol. 12, no. 2, pp. 1542-1552, 2005.

[5] D. J. Klionsky, "The molecular machinery of autophagy: unanswered questions," Journal of Cell Science, vol. 118, no. 1, pp. 7-18, 2005.

[6] K. Suzuki and Y. Ohsumi, "Molecular machinery of autophagosome formation in yeast, Saccharomyces cerevisiae," FEBS Letters, vol. 581, no. 11, pp. 2156-2161, 2007.

[7] B. Levine and G. Kroemer, "Autophagy in the pathogenesis of disease," Cell, vol. 132, no. 1, pp. 27-42, 2008.

[8] D. J. Klionsky and S. D. Emr, "Autophagy as a regulated pathway of cellular degradation," Science, vol. 290, no. 5497, pp. 1717-1721, 2000.

[9] A. M. Cuervo, "Autophagy: in sickness and in health," Trends in Cell Biology, vol. 14, no. 2, pp. 70-77, 2004.

[10] C. T. Chu, "Eaten alive: autophagy and neuronal cell death after hypoxia-ischemia," American Journal of Pathology, vol. 172, no. 2, pp. 284-287, 2008. 
[11] A. Meléndez, Z. Tallóczy, M. Seaman, E. L. Eskelinen, D. H. Hall, and B. Levine, "Autophagy genes are essential for dauer development and life-span extension in C. elegans," Science, vol. 301, no. 5638, pp. 1387-1391, 2003.

[12] T. Yorimitsu, S. Zaman, J. R. Broach, and D. J. Klionsky, "Protein kinase A and Sch9 cooperatively regulate induction of autophagy in Saccharomyces cerevisiae," Molecular Biology of the Cell, vol. 18, no. 10, pp. 4180-4189, 2007.

[13] T. Noda and Y. Ohsumi, "Tor, a phosphatidylinositol kinase homologue, controls autophagy in yeast," Journal of Biological Chemistry, vol. 273, no. 7, pp. 3963-3966, 1998.

[14] J. Liang, S. H. Shao, Z. X. Xu et al., "The energy sensing LKB1-AMPK pathway regulates p27kip1 phosphorylation mediating the decision to enter autophagy or apoptosis," Nature Cell Biology, vol. 9, no. 2, pp. 218-224, 2007.

[15] M. Lippai, G. Csikós, P. Maróy, T. Lukácsovich, G. Juhász, and M. Sass, "SNF4A $\gamma$, the Drosophila AMPK $\gamma$ subunit is required for regulation of developmental and stress-induced autophagy," Autophagy, vol. 4, no. 4, pp. 476-486, 2008.

[16] P. Syntichaki, K. Troulinaki, and N. Tavernarakis, "eIF4E function in somatic cells modulates ageing in Caenorhabditis elegans," Nature, vol. 445, no. 7130, pp. 922-926, 2007.

[17] M. Hansen, S. Taubert, D. Crawford, N. Libina, S. J. Lee, and C. Kenyon, "Lifespan extension by conditions that inhibit translation in Caenorhabditis elegans," Aging Cell, vol. 6, no. 1, pp. 95-110, 2007.

[18] K. Z. Pan, J. E. Palter, A. N. Rogers et al., "Inhibition of mRNA translation extends lifespan in Caenorhabditis elegans," Aging Cell, vol. 6, no. 1, pp. 111-119, 2007.

[19] N. Tavernarakis, "Ageing and the regulation of protein synthesis: a balancing act?" Trends in Cell Biology, vol. 18, no. 5, pp. 228-235, 2008.

[20] R. Scherz-Shouval and Z. Elazar, "ROS, mitochondria and the regulation of autophagy," Trends in Cell Biology, vol. 17, no. 9, pp. 422-427, 2007.

[21] P. Codogno and A. J. Meijer, "Autophagy and signaling: their role in cell survival and cell death," Cell Death and Differentiation, vol. 12, supplement 2, pp. 1509-1518, 2005.

[22] D. Crighton, S. Wilkinson, J. O'Prey et al., "DRAM, a p53induced modulator of autophagy, is critical for apoptosis," Cell, vol. 126, no. 1, pp. 121-134, 2006.

[23] E. Tasdemir, M. C. Maiuri, L. Galluzzi et al., "Regulation of autophagy by cytoplasmic p53," Nature Cell Biology, vol. 10, no. 6, pp. 676-687, 2008.

[24] S. Kumar and D. Cakouros, "Transcriptional control of the core cell-death machinery," Trends in Biochemical Sciences, vol. 29, no. 4, pp. 193-199, 2004.

[25] S. Polager, M. Ofir, and D. Ginsberg, "E2F1 regulates autophagy and the transcription of autophagy genes," Oncogene, vol. 27, no. 35, pp. 4860-4864, 2008.

[26] J. Zhao, J. J. Brault, A. Schild et al., "FoxO3 coordinately activates protein degradation by the autophagic/lysosomal and proteasomal pathways in atrophying muscle cells," Cell Metabolism, vol. 6, no. 6, pp. 472-483, 2007.

[27] S. I. Imai, C. M. Armstrong, M. Kaeberlein, and L. Guarente, "Transcriptional silencing and longevity protein Sir2 is an NAD-dependent histone deacetylase," Nature, vol. 403, no. 6771, pp. 795-800, 2000.

[28] H. A. Tissenbaum and L. Guarente, "Increased dosage of a sir-2 gene extends lifespan in Caenorhabditis elegans," Nature, vol. 410, no. 6825, pp. 227-230, 2001.

[29] M. Kaeberlein, M. McVey, and L. Guarente, "The SIR2/3/4 complex and SIR2 alone promote longevity in Saccharomyces cerevisiae by two different mechanisms," Genes and Development, vol. 13, no. 19, pp. 2570-2580, 1999.
[30] J. G. Wood, B. Rogina, S. Lavu et al., "Sirtuin activators mimic caloric restriction and delay ageing in metazoans," Nature, vol. 430, no. 7000, pp. 686-689, 2004.

[31] H. L. In, L. Cao, R. Mostoslavsky et al., "A role for the NADdependent deacetylase Sirt1 in the regulation of autophagy," Proceedings of the National Academy of Sciences of the United States of America, vol. 105, no. 9, pp. 3374-3379, 2008.

[32] A. Massey, C. Zhang, and A. Cuervo, "Chaperone-mediated autophagy in aging and disease," Current Topics in Developmental Biology, vol. 73, pp. 205-235, 2006.

[33] J. F. Dice, "Peptide sequences that target cytosolic proteins for lysosomal proteolysis," Trends in Biochemical Sciences, vol. 15, no. 8, pp. 305-309, 1990.

[34] H. L. Chiang, S. R. Terlecky, C. P. Plant, and J. F. Dice, "A role for a 70-kilodaton heat shock protein in lysosomal degradation of intracellular proteins," Science, vol. 246, no. 4928, pp. 382-385, 1989.

[35] A. L. Kovács, Z. Pálfia, G. Réz, T. Vellai, and J. Kovács, "Sequestration revisited: Integrating traditional electron microscopy, de novo assembly and new results," Autophagy, vol. 3, no. 6, pp. 655-662, 2007.

[36] A. Majesk and J. Dice, "Mechanisms of chaperone-mediated autophagy," The International Journal of Biochemistry \& Cell Biology, vol. 36, pp. 2435-2444, 2004.

[37] A. M. Cuervo and J. F. Dice, "A receptor for the selective uptake and degradation of proteins by lysosomes," Science, vol. 273, no. 5274, pp. 501-503, 1996.

[38] N. Salvador, C. Aguado, M. Horst, and E. Knecht, "Import of a cytosolic protein into lysosomes by chaperone-mediated autophagy depends on its folding state," Journal of Biological Chemistry, vol. 275, no. 35, pp. 27447-27456, 2000.

[39] F. A. Agarraberes, S. R. Terlecky, and J. F. Dice, "An intralysosomal hsp70 is required for a selective pathway of lysosomal protein degradation," Journal of Cell Biology, vol. 137, no. 4, pp. 825-834, 1997.

[40] S. Terlecky and J. Dice, "Polypeptide import and degradation by isolated lysosomes," The Journal of Biological Chemistry, vol. 268, pp. 23490-23495, 1993.

[41] F. Aniento, A. G. Papavassiliou, E. Knecht, and E. Roche, "Selective uptake and degradation of c-Fos and v-Fos by rat liver lysosomes," FEBS Letters, vol. 390, no. 1, pp. 47-52, 1996.

[42] S. S. Wing, H. L. Chiang, A. L. Goldberg, and J. F. Dice, "Proteins containing peptide sequences related to Lys-PheGlu-Arg-Gln are selectively depleted in liver and heart, but not skeletal muscle, of fasted rats," Biochemical Journal, vol. 275, no. 1, pp. 165-169, 1991.

[43] A. M. Cuervo, E. Knecht, S. R. Terlecky, and J. F. Dice, "Activation of a selective pathway of lysosomal proteolysis in rat liver by prolonged starvation," American Journal of Physiology, vol. 269, no. 5, pp. C1200-C1208, 1995.

[44] R. Kiffin, C. Christian, E. Knecht, and A. M. Cuervo, "Activation of chaperone-mediated autophagy during oxidative stress," Molecular Biology of the Cell, vol. 15, no. 11, pp. 48294840, 2004.

[45] A. M. Cuervo, H. Hildebrand, E. M. Bomhard, and J. F. Dice, "Direct lysosomal uptake of $\alpha 2$-microglobulin contributes to chemically induced nephropathy," Kidney International, vol. 55, no. 2, pp. 529-545, 1999.

[46] D. Hanahan and R. A. Weinberg, "The hallmarks of cancer," Cell, vol. 100, no. 1, pp. 57-70, 2000. 
[47] J. C. Reed, "Mechanisms of apoptosis," The American Journal of Pathology, vol. 39, pp. 1415-1430, 2000.

[48] H. S. Kim, J. W. Lee, Y. H. Soung et al., "Inactivating mutations of caspase-8 gene in colorectal carcinomas," Gastroenterology, vol. 125, no. 3, pp. 708-715, 2003.

[49] J. W. Lee, Y. H. Soung, S. Y. Kim et al., "Inactivating mutations of proapoptotic Bad gene in human colon cancers," Carcinogenesis, vol. 25, no. 8, pp. 1371-1376, 2004.

[50] S. H. Lee, M. S. Shin, W. S. Park et al., "Alterations of Fas (Apo-1/CD95) gene in non-small cell lung cancer," Oncogene, vol. 18, no. 25, pp. 3754-3760, 1999.

[51] M. S. Shin, H. S. Kim, C. S. Kang et al., "Inactivating mutations of CASP10 gene in non-Hodgkin lymphomas," Blood, vol. 99, no. 11, pp. 4094-4099, 2002.

[52] M. R. Kang, M. S. Kim, J. E. Oh et al., "Frameshift mutations of autophagy-related genes ATG2B, ATG5, ATG9B and ATGI2 in gastric and colorectal cancers with microsatellite instability," Journal of Pathology, vol. 217, no. 5, pp. 702-706, 2009.

[53] M. S. Kim, E. G. Jeong, C. H. Ahn, S. S. Kim, S. H. Lee, and N. J. Yoo, "Frameshift mutation of UVRAG, an autophagyrelated gene, in gastric carcinomas with microsatellite instability," Human Pathology, vol. 39, no. 7, pp. 1059-1063, 2008.

[54] J. W. Lee, E. G. Jeong, S. H. Lee, N. J. Yoo, and S. H. Lee, "Somatic mutations of BECN1, an autophagy-related gene, in human cancers," APMIS, vol. 115, no. 6, pp. 750-756, 2007.

[55] V. M. Aita, X. H. Liang, V. V. V. S. Murty et al., "Cloning and genomic organization of beclin 1, a candidate tumor suppressor gene on chromosome 17q21," Genomics, vol. 59, no. 1, pp. 59-65, 1999.

[56] J. Marx, "Autophagy: is it cancer's friend or foe?" Science, vol. 312, no. 5777, pp. 1160-1161, 2006.

[57] J. Thorburn, F. Moore, A. Rao et al., "Selective inactivation of a Fas-associated death domain protein (FADD)-dependent apoptosis and autophagy pathway in immortal epithelial cells," Molecular Biology of the Cell, vol. 16, no. 3, pp. 11891199, 2005.

[58] K. R. Mills, M. Reginato, J. Debnath, B. Queenan, and J. S. Brugge, "Tumor necrosis factor-related apoptosis-inducing ligand (TRAIL) is required for induction of autophagy during lumen formation in vitro," Proceedings of the National Academy of Sciences of the United States of America, vol. 101, no. 10, pp. 3438-3443, 2004.

[59] E. M. Hammond, C. L. Brunet, G. D. Johnson et al., "Homology between a human apoptosis specific protein and the product of APG5, a gene involved in autophagy in yeast," FEBS Letters, vol. 425, no. 3, pp. 391-395, 1998.

[60] D. J. Klionsky, "Autophagy: from phenomenology to molecular understanding in less than a decade," Nature Reviews Molecular Cell Biology, vol. 8, no. 11, pp. 931-937, 2007.

[61] I. Tanida, E. Tanida-Miyake, M. Komatsu, T. Ueno, and E. Kominami, "Human Apg3p/Autlp homologue is an authentic $\mathrm{E}_{2}$ enzyme for multiple substrates, GATE-16, GABARAP, and MAP-LC3, and facilitates the conjugation of hApg12p to hApg5p," Journal of Biological Chemistry, vol. 277, no. 16, pp. 13739-13744, 2002.

[62] S. Yousefi, R. Perozzo, I. Schmid et al., "Calpain-mediated cleavage of Atg5 switches autophagy to apoptosis," Nature Cell Biology, vol. 8, no. 10, pp. 1124-1132, 2006.

[63] P. Boya, R. A. Gonzalez-Polo, N. Casares et al., "Inhibition of macroautophagy triggers apoptosis," Molecular and Cellular Biology, vol. 25, pp. 1025-1040, 2005.
[64] J. J. Lum, D. E. Bauer, M. Kong et al., "Growth factor regulation of autophagy and cell survival in the absence of apoptosis," Cell, vol. 120, no. 2, pp. 237-248, 2005.

[65] Z. Yue, S. Jin, C. Yang, A. J. Levine, and N. Heintz, "Beclin 1 , an autophagy gene essential for early embryonic development, is a haploinsufficient tumor suppressor," Proceedings of the National Academy of Sciences of the United States of America, vol. 100, no. 25, pp. 15077-15082, 2003.

[66] X. Qu, J. Yu, G. Bhagat et al., "Promotion of tumorigenesis by heterozygous disruption of the beclin 1 autophagy gene," The Journal of Clinical Investigation, vol. 112, pp. 1809-1820, 2003.

[67] Y. Kondo, T. Kanzawa, R. Sawaya, and S. Kondo, "The role of autophagy in cancer development and response to therapy," Nature Reviews Cancer, vol. 5, no. 9, pp. 726-734, 2005.

[68] Z. Feng, H. Zhang, A. J. Levine, and S. Jin, "The coordinate regulation of the p53 and mTOR pathways in cells," Proceedings of the National Academy of Sciences of the United States of America, vol. 102, no. 23, pp. 8204-8209, 2005.

[69] S. Arico, A. Petiot, C. Bauvy et al., "The tumor suppressor PTEN positively regulates macroautophagy by inhibiting the phosphatidylinositol 3-kinase/protein kinase B pathway," Journal of Biological Chemistry, vol. 276, no. 38, pp. 3524335246, 2001.

[70] S. Pattingre, A. Tassa, X. Qu et al., "Bcl-2 antiapoptotic proteins inhibit Beclin 1-dependent autophagy," Cell, vol. 122, no. 6, pp. 927-939, 2005.

[71] L. Yu, F. Wan, S. Dutta et al., "Autophagic programmed cell death by selective catalase degradation," Proceedings of the National Academy of Sciences of the United States of America, vol. 103, no. 13, pp. 4952-4957, 2006.

[72] T. Shintani and D. J. Klionsky, "Autophagy in health and disease: a double-edged sword," Science, vol. 306, no. 5698, pp. 990-995, 2004.

[73] N. Mizushima, A. Yamamoto, M. Matsui, T. Yoshimori, and Y. Ohsumi, "In vivo analysis of autophagy in response to nutrient starvation using transgenic mice expressing a fluorescent autophagosome marker," Molecular Biology of the Cell, vol. 15, no. 3, pp. 1101-1111, 2004.

[74] D. Kaneda, K. Sugie, A. Yamamoto et al., "A novel form of autophagic vacuolar myopathy with late-onset and multiorgan involvement," Neurology, vol. 61, no. 1, pp. 128-131, 2003.

[75] I. Nishino, M. C. V. Malicdan, K. Murayama, I. Nonaka, Y. K. Hayashi, and S. Noguchi, "Molecular pathomechanism of distal myopathy with rimmed vacuoles," Acta Myologica, vol. 24, no. 2, pp. 80-83, 2005.

[76] I. Nonaka, N. Sunohara, S. Ishiura, and E. Satoyoshi, "Familial distal myopathy with rimmed vacuole and lamellar (myeloid) body formation," Journal of the Neurological Sciences, vol. 51, no. 1, pp. 141-155, 1981.

[77] M. M. Hippert, P. S. O’Toole, and A. Thorburn, “Autophagy in cancer: good, bad, or both?” Cancer Research, vol. 66, no. 19, pp. 9349-9351, 2006.

[78] C. Yan, M. Tanaka, K. Sugie et al., "A new congenital form of X-linked autophagic vacuolar myopathy," Neurology, vol. 65, no. 7, pp. 1132-1134, 2005.

[79] M. C. V. Malicdan, S. Noguchi, and I. Nishino, "Autophagy in a mouse model of distal myopathy with rimmed vacuoles or hereditary inclusion body myopathy," Autophagy, vol. 3, no. 4, pp. 396-398, 2007.

[80] I. Nishino, J. Fu, K. Tanji et al., "Primary LAMP-2 deficiency causes X-linked vacoular cardiomyopathy and myopathy 
(Danon disease)," Nature, vol. 406, no. 6798, pp. 906-910, 2000.

[81] M. J. Danon, S. J. Oh, S. DiMauro et al., "Lysosomal glycogen storage disease with normal acid maltase," Neurology, vol. 31, no. 1, pp. 51-57, 1981.

[82] I. Nishino, "Autophagic vacuolar myopathies," Current Neurology and Neuroscience Reports, vol. 3, pp. 64-69, 2003.

[83] J. F. Dice, "Chaperone-mediated autophagy," Autophagy, vol. 3, no. 4, pp. 295-299, 2007.

[84] A. C. Massey, C. Zhang, and A. M. Cuervo, "Chaperonemediated autophagy in aging and disease," Current Topics in Developmental Biology, vol. 73, pp. 205-235, 2006.

[85] Y. Tanaka, G. Guhde, A. Suter et al., "Accumulation of autophagic vacuoles and cardiomyopathy LAMP-2-deficient mice," Nature, vol. 406, no. 6798, pp. 902-906, 2000.

[86] H. Kalimo, M. L. Savontaus, H. Lang et al., "X-linked myopathy with excessive autophagy: a new hereditary muscle disease," Annals of Neurology, vol. 23, no. 3, pp. 258-265, 1988.

[87] J. P. Louboutin, M. Villanova, B. Lucas-Héron, and M. Fardeau, "X-linked vacuolated myopathy: membrane attack complex deposition on muscle fiber membranes with calcium accumulation on sarcolemma," Annals of Neurology, vol. 41, no. 1, pp. 117-120, 1997.

[88] M. Villanova, J. P. Louboutin, D. Chateau et al., "Xlinked vacuolated myopathy: complement membrane attack complex on surface membrane of injured muscle fibers," Annals of Neurology, vol. 37, no. 5, pp. 637-645, 1995.

[89] C. Yan, M. Tanaka, K. Sugie et al., "A new congenital form of X-linked autophagic vacuolar myopathy," Neurology, vol. 65, no. 7, pp. 1132-1134, 2005.

[90] N. Raben, P. Plotz, and B. J. Byrne, "Acid $\alpha$-glucosidase deficiency (glycogenosis type II, pompe disease)," Current Molecular Medicine, vol. 2, no. 2, pp. 145-166, 2002.

[91] J. Smith, H. Zellweger, and A. K. Afifi, "Muscular form of glycogenosis, type II (Pompe)," Neurology, vol. 17, no. 6, pp. 537-549, 1967.

[92] M. L. C. Hagemans, L. P. F. Winkel, P. A. Van Doorn et al., "Clinical manifestation and natural course of late-onset Pompe's disease in 54 Dutch patients," Brain, vol. 128, no. 3, pp. 671-677, 2005.

[93] M. C. Dalakas, "Sporadic inclusion body myositisdiagnosis, pathogenesis and therapeutic strategies," Nature Clinical Practice Neurology, vol. 2, no. 8, pp. 437-447, 2006.

[94] V. Askanas and W. K. Engel, "Inclusion-body myositis: muscle-fiber molecular pathology and possible pathogenic significance of its similarity to Alzheimer's and Parkinson's disease brains," Acta Neuropathologica, vol. 116, no. 6, pp. 583-595, 2008.

[95] V. Askanas, W. K. Engel, and A. Nogalska, "Inclusion body myositis: a degenerative muscle disease associated with intra-muscle fiber multi-protein aggregates, proteasome inhibition, endoplasmic reticulum stress and decreased lysosomal degradation: mini-symposium: protein aggregate myopathies," Brain Pathology, vol. 19, no. 3, pp. 493-506, 2009.

[96] Y. Tsuruta, A. Furuta, K. Furuta, T. Yamada, J. I. Kira, and T. Iwaki, "Expression of the lysosome-associated membrane proteins in myopathies with rimmed vacuoles," Acta Neuropathologica, vol. 101, no. 6, pp. 579-584, 2001.

[97] A. M. Cuervo, L. Stafanis, R. Fredenburg, P. T. Lansbury, and D. Sulzer, "Impaired degradation of mutant $\alpha$-synuclein by chaperone-mediated autophagy," Science, vol. 305, no. 5688, pp. 1292-1295, 2004.
[98] A. M. Cuervo, S. R. Terlecky, J. F. Dice, and E. Knecht, "Selective binding and uptake of ribonuclease $\mathrm{A}$ and glyceraldehyde-3- phosphate dehydrogenase by isolated rat liver lysosomes," Journal of Biological Chemistry, vol. 269, no. 42, pp. 26374-26380, 1994.

[99] A. M. Cuervo, J. F. Dice, and E. Knecht, "A population of rat liver lysosomes responsible for the selective uptake and degradation of cytosolic proteins," Journal of Biological Chemistry, vol. 272, no. 9, pp. 5606-5615, 1997.

[100] F. Aniento, E. Roche, A. M. Cuervo, and E. Knecht, "Uptake and degradation of glyceraldehyde-3-phosphate dehydrogenase by rat liver lysosomes," Journal of Biological Chemistry, vol. 268, no. 14, pp. 10463-10470, 1993.

[101] W. K. Engel and V. Askanas, "Inclusion-body myositis: clinical, diagnostic, and pathologic aspects," Neurology, vol. 66, no. 2, pp. S20-S29, 2006.

[102] T. Kumamoto, H. Ueyama, H. Tsumura, I. Toyoshima, and T. Tsuda, "Expression of lysosome-related proteins and genes in the skeletal muscles of inclusion body myositis," Acta Neuropathologica, vol. 107, no. 1, pp. 59-65, 2004.

[103] G. Bjørkøy, T. Lamark, A. Brech et al., "p62/SQSTM1 forms protein aggregates degraded by autophagy and has a protective effect on huntingtin-induced cell death," Journal of Cell Biology, vol. 171, no. 4, pp. 603-614, 2005.

[104] M. L. Seibenhener, J. R. Babu, T. Geetha, H. C. Wong, N. R. Krishna, and M. W. Wooten, "Sequestosome 1/p62 is a polyubiquitin chain binding protein involved in ubiquitin proteasome degradation," Molecular and Cellular Biology, vol. 24, no. 18, pp. 8055-8068, 2004.

[105] A. Nogalska, C. Terracciano, C. D’Agostino, W. King Engel, and V. Askanas, "p62/SQSTM1 is overexpressed and prominently accumulated in inclusions of sporadic inclusion-body myositis muscle fibers, and can help differentiating it from polymyositis and dermatomyositis," Acta Neuropathologica, vol. 118, no. 3, pp. 407-413, 2009.

[106] J. N. Keller, K. B. Hanni, and W. R. Markesbery, "Impaired proteasome function in Alzheimer's disease," Journal of Neurochemistry, vol. 75, pp. 436-439, 2000.

[107] W. X. Ding and X. M. Yin, "Sorting, recognition and activation of the misfolded protein degradation pathways through macroautophagy and the proteasome," Autophagy, vol. 4, no. 2, pp. 141-150, 2008.

[108] S. Oddo, "The ubiquitin-proteasome system in Alzheimer's disease," Journal of Cellular and Molecular Medicine, vol. 12, pp. 363-373, 2008.

[109] M. Martinez-Vicente and A. M. Cuervo, "Autophagy and neurodegeneration: when the cleaning crew goes on strike," Lancet Neurology, vol. 6, no. 4, pp. 352-361, 2007.

[110] R. A. Nixon, "Autophagy, amyloidogenesis and Alzheimer disease," Journal of Cell Science, vol. 120, no. 23, pp. 40814091, 2007.

[111] J. J. Shacka, K. A. Roth, and J. Zhang, "The autophagylysosomal degradation pathway: role in neurodegenerative disease and therapy," Frontiers in Bioscience, vol. 13, no. 2, pp. 718-736, 2008.

[112] R. A. Nixon, "Autophagy in neurodegenerative disease: friend, foe or turncoat?" Trends in Neurosciences, vol. 29, no. 9, pp. 528-535, 2006.

[113] M. Badadani, A. Nalbandian, G. D. Watts et al., "VCP associated inclusion body myopathy and paget disease of bone knock-in mouse model exhibits tissue pathology typical of human disease," PLoS ONE, vol. 5, no. 10, article e13183, 2010 . 
[114] W. Bursch, "The autophagosomal-lysosomal compartment in programmed cell death," Cell Death and Differentiation, vol. 8, no. 6, pp. 569-581, 2001.

[115] P. O. Seglen and P. B. Gordon, "3-Methyladenine: specific inhibitor of autophagic/lysosomal protein degradation in isolated rat hepatocytes," Proceedings of the National Academy of Sciences of the United States of America, vol. 79, no. 6, pp. 1889-1892, 1982.

[116] E. J. Bowman, A. Siebers, and K. Altendorf, "Bafilomycins: a class of inhibitors of membrane ATPases from microorganisms, animal cells, and plant cells," Proceedings of the National Academy of Sciences of the United States of America, vol. 85, no. 21, pp. 7972-7976, 1988.

[117] K. Yamanaka, Y. Sasagawa, and T. Ogura, "Recent advances in p97/VCP/Cdc48 cellular functions," Biochimica et Biophysica Acta, vol. 1823, no. 1, pp. 130-137, 2011.

[118] R. B. Shirley, I. Kaddour-Djebbar, D. M. Patel, V. Lakshmikanthan, R. W. Lewis, and M. V. Kumar, "Combination of proteasomal inhibitors lactacystin and MG132 induced synergistic apoptosis in prostate cancer cells," Neoplasia, vol. 7, no. 12, pp. 1104-1111, 2005. 

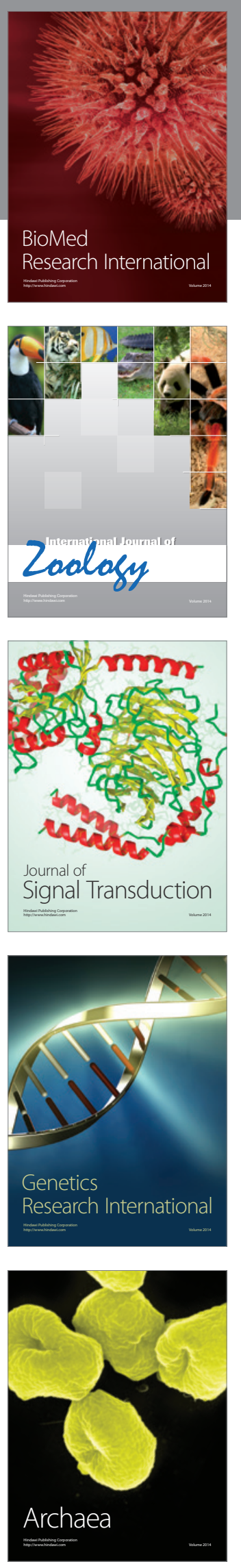
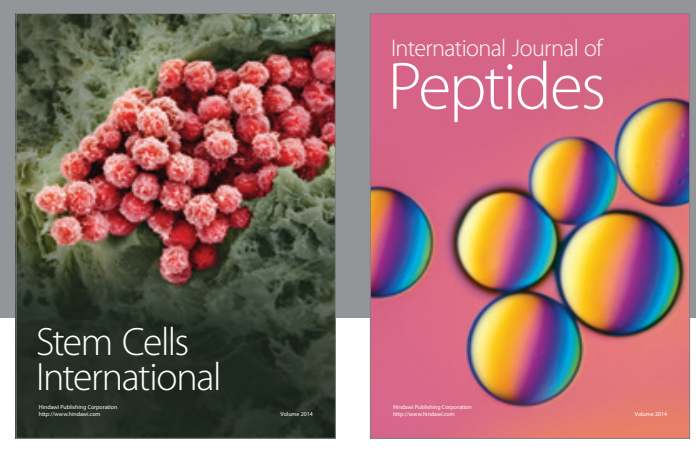

Submit your manuscripts at

http://www.hindawi.com
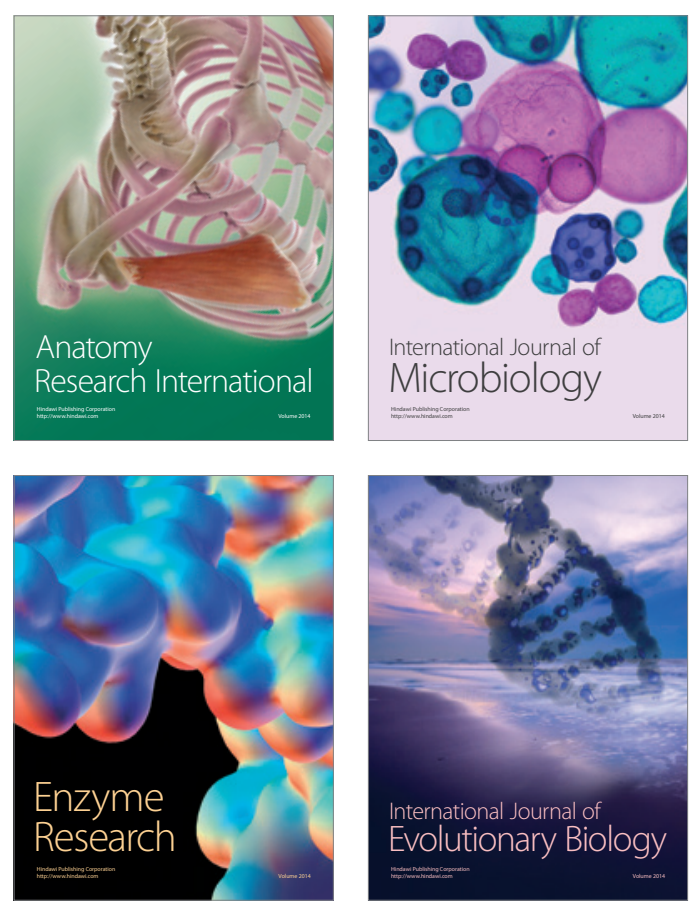
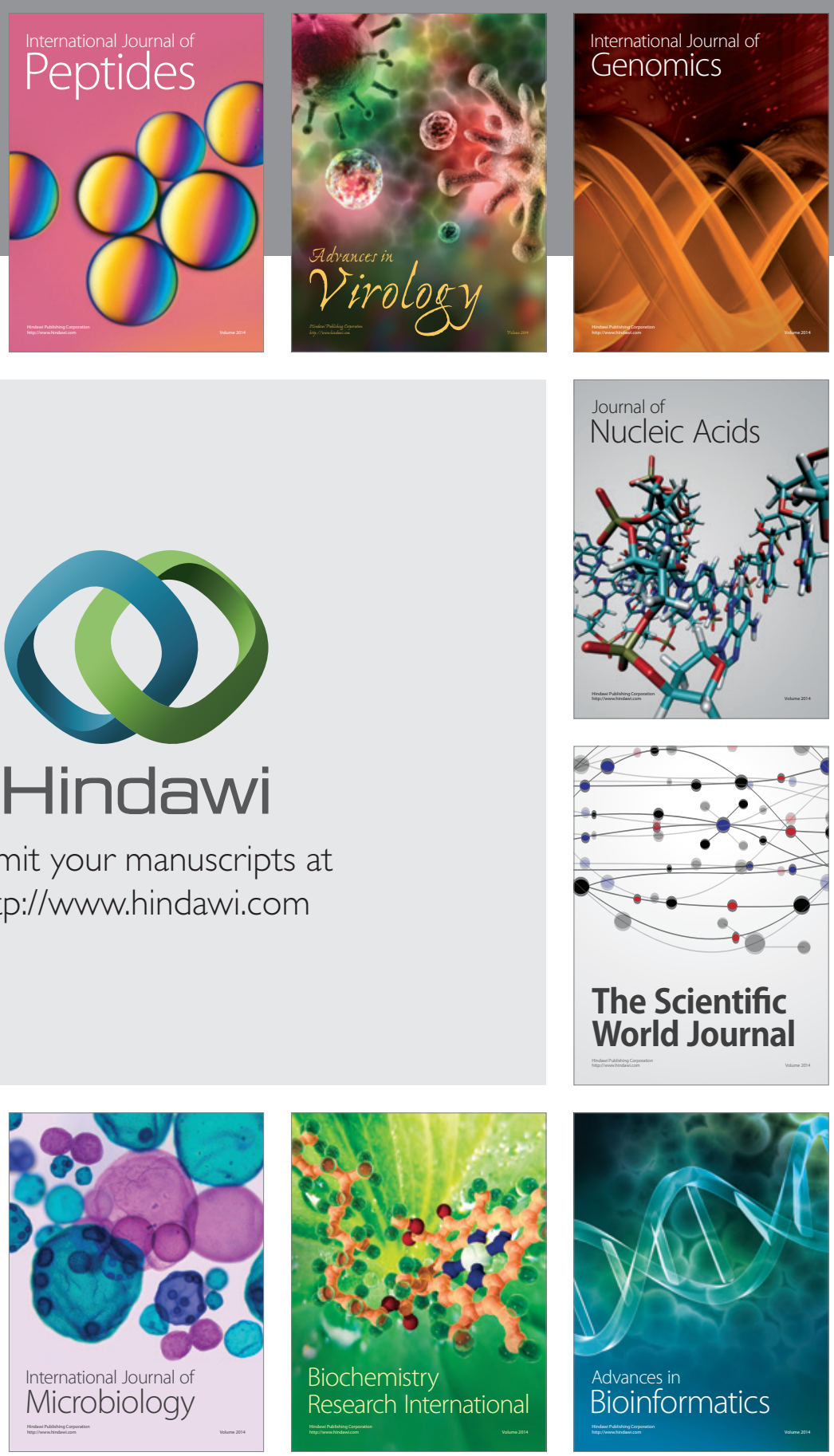

The Scientific World Journal
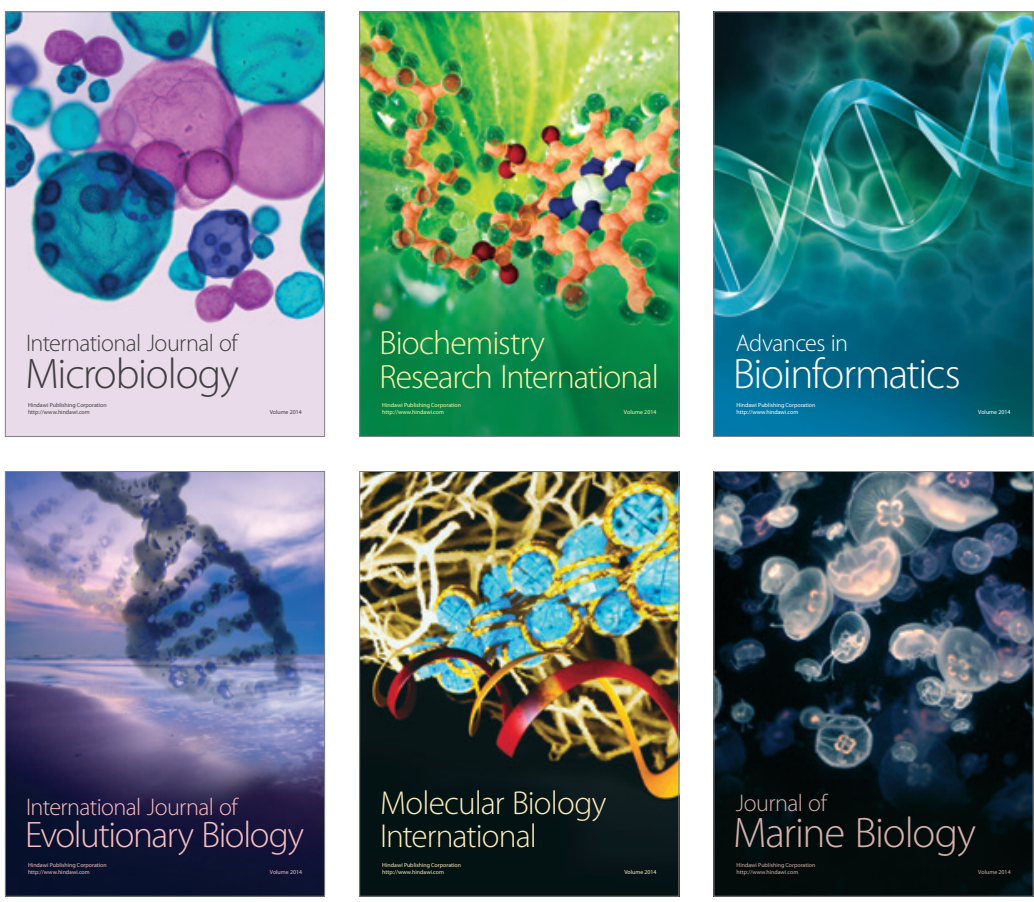